Chem. Ber. 115, $1089-1102$ (1982)

\title{
Die Fries-Umlagerung als Gleichgewichtsreaktion ${ }^{1)}$
}

\author{
Franz Effenberger* und Rainer Gutmann ${ }^{2)}$
}

Institut für Organische Chemie der Universität Stuttgart, Pfaffenwaldring 55, D-7000 Stuttgart 80

Eingegangen am 1. Juli 1981

Die Fries-Umlagerung von Arylbenzoaten 1 - in 1,2-Dichlorethan mittels Trifluormethansulfonsäure als Katalysator - zu (ortho- und para-Hydroxyaryl)phenylketonen 2 bzw. 3 ist bei $170^{\circ} \mathrm{C}$ reversibel und führt zu Gleichgewichtsgemischen aus 1, 2 und 3. Das Gleichgewicht wird, von den Arylestern 1 und den Hydroxyaryl-ketonen 2 bzw. 3 ausgehend, gleichermaßen eingestellt. Die größere Stabilität der ortho- gegenüber den para-Hydroxyaryl-ketonen kann aus den über ${ }^{13} \mathrm{C}$ NMR-Spektren zugänglichen Verdrillungswinkeln $\Theta$ zwischen der Carbonylgruppe und dem aromatischen $\pi$-System zufriedenstellend erklärt werden. Für die Acylgruppenwanderung wird ein dissoziativer Mechanismus vorgeschlagen.

\section{The Fries Rearrangement as an Equilibrium Reaction 1)}

The Fries rearrangement of arylbenzoates 1, in 1,2-dichloroethane in the presence of trifluoromethanesulfonic acid (TFMS) as catalyst, to ortho- and para-hydroxyaryl ketones ( 2 and 3 , resp.) is reversible at $170^{\circ} \mathrm{C}$; an equilibrium of the compounds 1,2 , and 3 is established. The equilibrium is achieved starting from the aryl esters 1 as well as from the hydroxyaryl ketones 2 and 3, respectively. The higher stability of the ortho-compounds 2 compared to the para-compounds 3 can be explained by greater resonance interaction between the carbonyl group and the aromatic ring in 2 . The torsion angles $\Theta$, which are good indicators of this interaction, can be determined from ${ }^{13} \mathrm{C}$ NMR spectra. A dissociative mechanism is proposed for the acyl group migration.

Die Umlagerung von Arylestern unter dem Einfluß von Lewis- bzw. Brønsted-Säuren (FriesUmlagerung) ist zweifellos die wichtigste Methode zur Herstellung von Hydroxyaryl-ketonen ${ }^{3)}$. Da bei der Umlagerung von Phenylestern je nach eingesetztem Substrat und angewandten Reaktionsbedingungen isomere ortho- und para-Hydroxyphenyl-ketone in sehr unterschiedlicher Verteilung entstehen, wurden eine Reihe von Untersuchungen zum Mechanismus der Fries-Umlagerung durchgeführt mit dem Ziel, Erklärungen für die unterschiedliche Isomerenbildung zu finden.

Dewar und Hart $^{4)}$ kamen zu dem Schluß, daß bei den mit Aluminiumbromid durchgeführten Umsetzungen von Phenylbenzoat nebeneinander intramolekulare Umlagerung zum orthoHydroxyketon und intermolekulare Umlagerung zum ortho- und para-Hydroxyketon erfolgen. Die Autoren postulieren eine irreversible Bildung der Hydroxyaryl-ketone als notwendige Voraussetzung für die Deutung des Reaktionsablaufs aus der Isomerenverteilung. Daß die Fries-Umlagerung jedoch in bestimmten Fällen reversibel ist, konnten schon Rosenmund und Schnurr ${ }^{5)}$ bei der Umsetzung von 4-Acyl-3-alkylphenolen mit Brønsted-Säuren zeigen. Diese Befunde wurden zwar später angezweifelt ${ }^{6 a}$ ), konnten jedoch von Miquel et al. ${ }^{6 b}$ ) bestätigt werden.

(C) Verlag Chemie GmbH, D-6940 Weinheim, 1982

$0009-2940 / 82 / 0303-1089 \$ 02.50 / 0$ 
In einer Kurzmitteilung haben wir über die Fries-Umlagerung von Arylbenzoaten und die Retro-Fries-Umlagerung von ortho-Hydroxyaryl-ketonen in Gegenwart katalytischer Mengen Trifluormethansulfonsäure (TFMS) berichtet ${ }^{7}$. Nachdem aus den damaligen experimentellen Befunden nur beschränkte Aussagen möglich waren, ob es sich dabei um echte Gleichgewichtsreaktionen handelt, haben wir weitere umfangreiche experimentelle Untersuchungen zu diesem Problem durchgeführt, deren Ergebnisse und Folgerungen bezüglich des Mechanismus der Fries-Umlagerung Inhalt der vorliegenden Publikation sind.

\section{Umsetzungen von Arylbenzoaten 1 und (ortho- bzw. para-Hydroxyaryl)- phenylketonen 2 bzw. 3 mit TFMS unter äquilibrierenden Bedingungen}

Zur Bestimmung, ob es sich bei der Fries- bzw. Retro-Fries-Umlagerung unter bestimmten Bedingungen um Gleichgewichtsreaktionen handelt, setzten wir Arylbenzoate 1 und deren Friessche Umlagerungsprodukte, die (ortho- bzw. paraHydroxyaryl)phenylketone 2 bzw. 3, mit TFMS unter Variation von Reaktionszeit und -temperatur sowie der Säurekonzentration unter vergleichbaren Bedingungen um. Die Reaktionsparameter wurden so gewählt, daß möglichst wenig irreversible Nebenreaktionen erfolgen und die Gesamtausbeute an den Isomeren 1, 2 und 3 nicht unter $80 \%$ sank. Im Falle einer Gleichgewichtsreaktion müßten nach Erreichen des Gleichgewichtszustandes, jeweils ausgehend von den Reinverbindungen 1, 2 oder 3, gleiche Isomerenverteilungen resultieren.

Die literaturbekannten Arylbenzoate 1 und die (Hydroxyaryl)phenylketone 2 bzw. 3 wurden synthetisiert und gaschromatographisch auf Isomerenreinheit überprüft; die bislang noch nicht bekannten Verbindungen wurden auf eindeutigem Wege isomerenfrei dargestellt (s. exp. Teil). Es wurden jeweils $1.0 \mathrm{mmol}$ der reinen Verbindungen mit TFMS in absol. 1,2-Dichlorethan (DCE) als Lösungsmittel in verschlossenen Glasampullen unter Stickstoff thermostatisiert. Besondere Sorgfalt mußte hierbei auf die Reinigung des Lösungsmittels verwendet werden, da geringste Spuren an Verunreinigungen zu großen Anteilen an Zersetzungsprodukten führten. Nach Neutralisieren der Reaktionsgemische, Zugeben der jeweiligen Standardlösungen und wäßriger Aufarbeitung wurde die quantitative Zusammensetzung der erhaltenen Produktgemische gaschromatographisch bestimmt.

Isomerisierung von Phenylbenzoat (1 a), (2-Hydroxyphenyl)- (2a) und (4-Hydroxyphenyl)phenylketon (3a) in Gegenwart von TFMS

Die Umsetzungen der reinen Isomeren wurden bei $160^{\circ} \mathrm{C}$ unter Variation der Säurekonzentration sowie der Reaktionszeit durchgeführt. Als Produkte wurden neben den eingesetzten Verbindungen 1a, 2 a und 3a jeweils noch Phenol, [2-(Benzoyloxy)phenyl]phenylketon (4) und [4-(Benzoyloxy)phenyl]phenylketon (5) nachgewiesen; der Anteil an 4 lag jedoch unter der durch gaschromatographische Integration quantitativ bestimmbaren Menge $(<0.2 \%)$ (Tab. 1).

Die in Tab. 1 zusammengefaßten Ergebnisse beweisen, daß sowohl die Fries-Umlagerung von $1 \mathrm{a}$ als auch die Retro-Fries-Umlagerung von $\mathbf{2 a}$ bzw. 3a unter den angegebenen Bedingungen reversibel sind und zu Gleichgewichtsgemischen führen. Erwartungsgemäß erfolgt dabei die Gleichgewichtseinstellung bei höherer TFMS-Konzentration in 
<smiles>O=C(Oc1ccccc1)c1ccccc1</smiles>

1a<smiles>O=C(c1ccccc1)c1ccccc1O</smiles>

$\mathbf{2} \mathbf{a}$<smiles>O=C(c1ccccc1)c1ccc(O)cc1</smiles>

$3 \mathbf{a}$<smiles>O=C(Oc1ccccc1C(=O)c1ccccc1)c1ccccc1</smiles>

4<smiles>O=C(Oc1ccc(C(=O)c2ccccc2)cc1)c1ccccc1</smiles>

5

Tab. 1. Isomerisierung von $1 \mathbf{a}, 2 \mathrm{a}$ und $\mathbf{3 a}$ (jeweils $1.0 \mathrm{mmol}$ ) in Gegenwart von TFMS in absol. 1,2-Dichlorethan als Lösungsmittel bei $160^{\circ} \mathrm{C}$

\begin{tabular}{|c|c|c|c|c|c|c|c|c|c|}
\hline \multirow{2}{*}{ Substrat } & \multirow{2}{*}{$\begin{array}{l}\text { TFMS } \\
\text { mol- } \%\end{array}$} & \multirow{2}{*}{$\begin{array}{l}\text { Reakt.- } \\
\text { zeit h }\end{array}$} & \multicolumn{7}{|c|}{ Produkte Ausb. (\%) } \\
\hline & & & $1 \mathrm{a}$ & $2 \mathbf{a}$ & $3 \mathbf{a}$ & 4 & 5 & Phenol & \\
\hline $1 \mathrm{a}$ & 2 & 24 & 75.9 & 11.8 & 1.6 & 0.3 & 5.7 & 4.8 & \\
\hline $2 \mathbf{a}$ & 2 & 24 & 12.7 & 83.5 & 0.2 & 0.2 & 0.2 & 3.0 & \\
\hline 3a & 2 & 24 & 7.2 & 0.2 & 68.1 & 0.2 & 12.2 & 7.3 & \\
\hline $1 \mathbf{a}$ & 20 & 24 & 44.5 & 34.2 & 6.6 & 0.2 & 6.0 & 6.2 & \\
\hline $2 \mathbf{a}$ & 20 & 24 & 40.8 & 40.0 & 5.1 & 0.2 & 4.2 & 6.2 & \\
\hline 3a & 20 & 24 & 45.0 & 25.9 & 7.1 & 0.2 & 5.5 & 7.1 & \\
\hline $\mathbf{1 a}$ & 20 & 72 & 39.9 & 33.9 & 4.9 & 0.2 & 4.0 & $7.0)$ & \\
\hline $2 \mathbf{a}$ & 20 & 72 & 39.1 & 34.1 & 4.9 & 0.2 & 4.2 & 6.4 & Gleichgewicht \\
\hline $3 \mathbf{a}$ & 20 & 72 & 39.4 & 33.3 & 4.7 & 0.2 & 3.6 & 7.4 & \\
\hline $1 \mathbf{a}$ & 50 & 24 & 40.6 & 33.8 & 7.0 & 0.2 & 2.6 & 7.0 & \\
\hline $2 a$ & 50 & 24 & 40.2 & 33.6 & 6.9 & 0.2 & 3.0 & 6.4 & Gleichgewicht \\
\hline $3 \mathbf{a}$ & 50 & 24 & 40.2 & 33.6 & 6.9 & 0.2 & 2.4 & 7.3 & \\
\hline
\end{tabular}

kürzerer Zeit. Bei $160^{\circ} \mathrm{C}$ ist z. B. in Gegenwart von $20 \mathrm{~mol}-\%$ Säure nach $72 \mathrm{~h}$, in Gegenwart von $50 \mathrm{~mol}-\%$ Säure dagegen bereits nach $24 \mathrm{~h}$ das Gleichgewicht eingestellt. Die Bildung von Phenol und der Verbindungen 4 und 5 resultiert offensichtlich aus der Benzoylierung der Hydroxyketone 2a und 3a; hierfür sprechen auch die Ergebnisse der Isomerisierung von $3 \mathrm{a}$ in Gegenwart von 2 mol- \% TFMS, die nach $24 \mathrm{~h}$ bei $160^{\circ} \mathrm{C} 12 \%$ 5 und nur Spuren an 4 ergibt.

Isomerisierung von (3-Methylphenyl)benzoat (1b), (2-Hydroxy-4-methylphenyl)- (2b), (2-Hydroxy-6-methylphenyl)- ( $\left.2 b^{\prime}\right)$ und (4-Hydroxy-2-methylphenyl)-phenylketon (3b) in Gegenwart von TFMS

Nachdem Versuche zur Isomerisierung der meta-Kresolderivate $\mathbf{1 b}, \mathbf{2 b}$ und $\mathbf{3 b}$ in Gegenwart von 5 mol- $\%$ TFMS zwischen 150 und $170^{\circ} \mathrm{C}$ nach $72 \mathrm{~h}$ zu keiner Gleichgewichtseinstellung führten, untersuchten wir die Isomerisierung dieser Verbindungen in Gegenwart von $10 \mathrm{~mol}-\%$ TFMS bei $170^{\circ} \mathrm{C}$ in Abhängigkeit von der Reaktionszeit. Dabei erhielten wir nach $72 \mathrm{~h}$ ein Gleichgewicht, das neben wenig meta-Kresol ungefähr gleiche Anteile an 1b und $\mathbf{2 b}$ enthielt (Tab. 2). Das vierte mögliche Isomere, (2Hydroxy-6-methylphenyl)phenylketon ( $\mathbf{2}^{\prime}$ ) wurde in keinem Fall beobachtet. Die Isomerisierung von $\mathbf{2} \mathbf{b}^{\prime}$ unter vergleichenden Bedingungen führte jedoch ebenfalls nur zur 
Bildung von $\mathbf{1 b}$ und $\mathbf{2 b}$ neben wenig meta-Kresol. Allerdings weicht die Isomerenverteilung und vor allem die Gesamtausbeute von den mit $\mathbf{1 b}, \mathbf{2}$ b bzw. $\mathbf{3}$ b erhaltenen Ergebnissen deutlich $\mathrm{ab}$. Offensichtlich ist für diese Abweichung die Instabilität von $\mathbf{2} \mathbf{b}^{\prime}$ verantwortlich, was in verstärktem Maße zu Neben- und Zersetzungsreaktionen führt.<smiles>Cc1cccc(OC(=O)c2ccccc2)c1</smiles>

$\mathbf{1 b}$<smiles>Cc1ccc(C(=O)c2ccccc2)c(O)c1</smiles>

2b<smiles>Cc1cccc(O)c1C(=O)c1ccccc1</smiles>

$2 \mathbf{b}^{\prime}$<smiles>Cc1cc(O)ccc1C(=O)c1ccccc1</smiles>

3b

Tab. 2. Isomerisierung von $\mathbf{1 b}, \mathbf{2} \mathbf{b}, \mathbf{2} \mathbf{b}^{\prime}$ und $\mathbf{3 b}$ (jeweils $1.0 \mathrm{mmol}$ ) in Gegenwart von $10 \mathrm{~mol}-\%$ TFMS in absol. 1,2-Dichlorethan als Lösungsmittel bei $170^{\circ} \mathrm{C}$

\begin{tabular}{|c|c|c|c|c|c|c|}
\hline \multirow{2}{*}{ Substrat } & \multirow{2}{*}{$\begin{array}{c}\text { Reaktionszeit } \\
\mathrm{h}\end{array}$} & \multicolumn{5}{|c|}{ Produkte Ausb. $(\%)$} \\
\hline & & 1b & $2 \mathbf{b}$ & $\mathbf{3 b}$ & $m$-Kresol & \\
\hline 1b & 0.33 & 75.9 & 7.9 & - & 5.4 & \\
\hline 2b & 0.33 & 5.5 & 87.3 & 0.4 & 2.5 & \\
\hline 3b & 0.33 & 71.3 & 6.9 & 1.3 & 5.2 & \\
\hline 1b & 1 & 61.8 & 19.5 & - & 5.5 & \\
\hline $2 b$ & 1 & 18.2 & 69.7 & - & 3.3 & \\
\hline $3 \mathbf{b}$ & 1 & 60.3 & 22.1 & -- & 5.7 & \\
\hline 1b & 24 & 41.9 & 41.2 & - & 6.0 & \\
\hline $2 b$ & 24 & 39.2 & 39.3 & - & 5.3 & \\
\hline $3 \mathbf{b}$ & 24 & 39.6 & 40.0 & - & 5.4 & \\
\hline $\mathbf{1 b}$ & 72 & 39.8 & 39.5 & - & 5.9 & \\
\hline $2 \mathrm{~b}$ & 72 & 39.5 & 38.7 & - & $6.3\}$ & Gleichgewicht \\
\hline $3 \mathbf{b}$ & 72 & 38.4 & 39.0 & - & 6.5 & \\
\hline $2 \mathbf{b}^{\prime}$ & 6 & 42.4 & 6.6 & - & 10.7 & \\
\hline $2 \mathbf{b}^{\prime}$ & 72 & 26.9 & 21.7 & - & 7.0 & \\
\hline
\end{tabular}

Von besonderem Interesse war die Isomerisierung des para-Hydroxyketons $\mathbf{3 b}$, das unter den angegebenen Bedingungen völlig unerwartet in kürzester Zeit eine RetroFries-Umlagerung zum Ester $\mathbf{1}$ b eingeht. So enthielt z. B. bei der Isomerisierung von $\mathbf{3 b}$ das gebildete Reaktionsgemisch bereits nach 20 min nur noch $1.3 \%$ 3b und $71.3 \% \mathbf{1 b}$ (Tab. 2). Mit Verlängerung der Reaktionszeit nahmen dann die Anteile an $\mathbf{1 b}$ bis zur Gleichgewichtseinstellung wieder ab unter gleichzeitiger Zunahme an $\mathbf{2 b}$. Diese Ergebnisse sind mit den bei der zeitabhängigen Isomerisierung des Esters $\mathbf{1 b}$ erhaltenen Resultaten vergleichbar, so daß die Isomerisierung des para-Hydroxyketons $\mathbf{3} \mathbf{b}$ über eine schnelle Umlagerung zum Ester $1 \mathbf{b}$ und daraus langsamer die Fries-Umlagerung zum ortho-Hydroxyketon $\mathbf{2 b}$ erfolgt. Dieses ist gegenüber dem para-Derivat $\mathbf{3 b}$ offensichtlich stabiler und isomerisiert mit einer dem Ester 1b vergleichbaren Geschwindigkeit.

Isomerisierung von (3-Chlorphenyl)benzoat (1c), (4-Chlor-2-hydroxyphenyl)- (2c), (2-Chlor-6-hydroxyphenyl)- (2c') und (2-Chlor-4-hydroxyphenyl)-phenylketon (3c) in Gegenwart von TFMS

Die Isomerisierung von Verbindungen mit einem elektronenabziehenden Substituenten untersuchten wir am Beispiel der meta-Chlor-substituierten Verbindungen 1c, 2c, 
$2 \mathbf{c}^{\prime}$ und $3 \mathbf{c}$ bei $170^{\circ} \mathrm{C}$ unter Variation der TFMS-Konzentration und der Reaktionszeit. In Vorversuchen hatte sich gezeigt, daß die Chlor-substituierten Verbindungen in ihrem Reaktionsverhalten ungefähr mit dem der Methyl-substituierten Isomeren übereinstimmen: Die Verbindungen $\mathbf{2} \mathbf{c}^{\prime}$ und $\mathbf{3} \mathbf{c}$ gingen ebenso wie $\mathbf{2} \mathbf{b}^{\prime}$ und $\mathbf{3 b}$ in kürzester Zeit eine Retro-Fries-Umlagerung zum Ester 1c ein, während $1 \mathrm{c}$ und $2 \mathrm{c}$ langsamer und untereinander vergleichbar isomerisierten. Aus diesem Grund wurden zur Bestimmung der Gleichgewichtsbedingungen nur 1c und 2c eingesetzt. Hierbei wurde eine Gleichgewichtseinstellung nach $72 \mathrm{~h}$ in Gegenwart von $20 \mathrm{~mol}-0$ TFMS erreicht, das erhaltene Reaktionsgemisch enthielt neben ca. 5\% 3-Chlorphenol ca. $50 \%$ 1c und ca. $33 \% 2 \mathrm{c}$.<smiles>O=C(Oc1cccc(Cl)c1)c1ccccc1</smiles>

1c<smiles>O=C(c1ccccc1)c1ccc(Cl)cc1O</smiles>

$2 c$<smiles>O=C(c1ccccc1)c1c(O)cccc1Cl</smiles>

$2 \mathbf{c}^{\prime}$<smiles>O=C(c1ccccc1)c1ccc(O)cc1Cl</smiles>

$3 c$

Tab. 3. Isomerisierung von $1 \mathbf{c}, \mathbf{2 c}, \mathbf{2} \mathbf{c}^{\prime}$ und $\mathbf{3 c}$ (jeweils $1.0 \mathrm{mmol}$ ) in Gegenwart von TFMS in absol. 1,2-Dichlorethan als Lösungsmittel bei $170^{\circ} \mathrm{C}$

\begin{tabular}{|c|c|c|c|c|c|c|c|c|}
\hline \multirow[b]{2}{*}{ Substrat } & \multirow{2}{*}{$\begin{array}{l}\text { TFMS } \\
\text { mol- } \%\end{array}$} & \multirow{2}{*}{$\begin{array}{c}\text { Reakt.- } \\
\text { zeit } \\
\text { h }\end{array}$} & \multicolumn{6}{|c|}{ Produkte Ausb. (\%) } \\
\hline & & & $1 \mathrm{c}$ & $2 c$ & $2 \mathbf{c}^{\prime}$ & $3 c$ & $\begin{array}{l}\text { 3-Chlor- } \\
\text { phenol }\end{array}$ & \\
\hline $1 \mathrm{c}$ & 5 & 24 & 77.3 & 14.1 & - & _- & 5.8 & \\
\hline $2 c$ & 5 & 24 & 16.0 & 73.0 & - & - & - & \\
\hline $1 \mathrm{c}$ & 10 & 24 & 65.3 & 28.2 & - & _- & 3.7 & \\
\hline $2 c$ & 10 & 24 & 30.3 & 48.3 & - & - & - & \\
\hline $1 \mathrm{c}$ & 20 & 24 & 51.5 & 34.5 & - & - & 4.2 & \\
\hline $2 c$ & 20 & 24 & 47.7 & 31.6 & - & - & 5.1 & \\
\hline $1 \mathrm{c}$ & 20 & 72 & 50.2 & 32.8 & - & _- & $4.7)$ & \\
\hline $2 c$ & 20 & 72 & 51.5 & 33.9 & - & - & 5.5 & Cloighoruicht \\
\hline $\mathbf{2} \mathbf{c}^{\prime}$ & 20 & 72 & 50.0 & 33.4 & - & - & 5.0 & Gleichgewicht \\
\hline 3c & 20 & 72 & 50.3 & 33.2 & - & - & 4.8 & \\
\hline
\end{tabular}

Isomerisierung von (3,5-Dimethylphenyl)benzoat (1 d), (2-Hydroxy-4,6-dimethylphenyl)- (2d) und (4-Hydroxy-2,6-dimethylphenyl)-phenylketon ( $3 \mathrm{~d}$ ) in Gegenwart von TFMS

Die Untersuchungen zur Isomerisierung der elektronenreichen, in beiden meta-Stellungen zum Phenylsauerstoff substituierten Verbindungen 1d, 2d und 3d führten wir unter Variation der TFMS-Konzentration bei $170^{\circ} \mathrm{C}$ durch. Nach $24 \mathrm{~h}$ hatte sich in Gegenwart von nur 5 mol- $\%$ TFMS ein Gleichgewicht eingestellt, das neben geringen<smiles>Cc1cc(C)cc(OC(=O)c2ccccc2)c1</smiles>

1d<smiles>Cc1cc(C)c(C(=O)c2ccccc2)c(O)c1</smiles>

2d<smiles>Cc1cc(O)cc(C)c1C(=O)c1ccccc1</smiles>

3d

Chem. Ber. 115 (1982) 
Tab. 4. Isomerisierung von $1 \mathbf{d}, \mathbf{2 d}$ und $\mathbf{3 d}$ (jeweils $1.0 \mathrm{mmol}$ ) in Gegenwart von TFMS in absol. 1,2-Dichlorethan als Lösungsmittel bei $170^{\circ} \mathrm{C}$ nach $24 \mathrm{~h}$

\begin{tabular}{|c|c|c|c|c|c|c|}
\hline \multirow{2}{*}{ Substrat } & \multirow{2}{*}{$\begin{array}{l}\text { TFMS } \\
\text { mol- } \%\end{array}$} & \multicolumn{5}{|c|}{ Produkte Ausb. (\%) } \\
\hline & & $1 \mathrm{~d}$ & $2 d$ & 3d & $\begin{array}{l}\text { 3,5-Dimethyl } \\
\text { phenol }\end{array}$ & \\
\hline 1d & 30 & 90.2 & 0.5 & 0.3 & 2.5 & \\
\hline 2d & 30 & 80.5 & 0.3 & 0.2 & 4.3 & \\
\hline 1d & 15 & 90.9 & 0.3 & & 1.8 & \\
\hline 2d & 15 & 86.4 & 0.2 & & 3.0 & \\
\hline 1d & 10 & 86.4 & 0.3 & & 4.3 & \\
\hline $2 d$ & 10 & 82.9 & 0.3 & & 5.4 & \\
\hline $1 d$ & 5 & 83.5 & 0.5 & & $2.7)$ & \\
\hline 2d & 5 & 83.5 & 0.5 & & $2.2\}$ & Gleichgewicht \\
\hline 3d & 5 & 83.2 & 0.4 & & 4.2 & \\
\hline
\end{tabular}

Mengen des 2-Hydroxyketons 2d (ca. 0.5\%) und wenigen Prozenten 3,5-Dimethylphenol nurmehr den Ester 1d (ca. 83\%) enthielt (Tab. 4).

Die in Tab. 4 aufgeführten Versuchsergebnisse zeigen, daß im Falle der Dimethylsubstituierten Isomeren $\mathbf{1 d}, \mathbf{2} \mathbf{d}$ und $\mathbf{3 d}$ die Gleichgewichtszusammensetzung offensichtlich von der Stabilität des Esters 1d bestimmt wird.

\section{Konformation der untersuchten (ortho-bzw. para-Hydroxyaryl)phenylketone 2 und 3}

In unseren früheren Untersuchungen zur säurekatalysierten Fries-Umlagerung ${ }^{7}$ haben wir eine thermodynamisch kontrollierte Produktbildung postuliert, bei der im Falle vergleichbarer Stabilität von Ester und Hydroxyketon beide Verbindungen nebeneinander vorliegen, im Falle sterischer Hinderung bei den Hydroxyketonen sich jedoch die Produktbildung zugunsten der sterisch nicht gehinderten Ester verschiebt.

Wir haben jetzt versucht, den sterischen Einfluß der Substituenten auf die Produktzusammensetzung des Gleichgewichts bei der Fries- und Retro-Fries-Umlagerung quantitativ zu erfassen. Bekanntlich wird in Gegenwart raumerfüllender ortho-Substituenten die Carbonylgruppe aus der Ebene des substituierten Aromaten herausgedreht, wodurch eine Änderung der Mesomeriewechselwirkung zwischen den $\pi$-Elektronen des Aromaten und der Carbonylgruppe bewirkt wird. Unter der Annahme, daß Proportionalität zwischen der Resonanzwechselwirkung und dem Torsionswinkel $\Theta$ zwischen der Ebene des Aromaten und der der Carbonylgruppe besteht, sollte mit Hilfe physikalischer Meßmethoden, die auf Veränderungen im $\pi$-Elektronensystem ansprechen, dieser Torsionswinkel $\Theta$ bestimmt werden können. In Anlehnung an die an Acetophenonen durchgeführten Untersuchungen von Dhami und Stothers ${ }^{8)}$ haben wir die Torsionswinkel $\Theta$ der Hydroxyketone $\mathbf{2}$ und $\mathbf{3}$ aus den chemischen Verschiebungen ihrer ${ }^{13} \mathrm{C}$-NMR-Spektren bestimmt und hierfür das zur Carbonylgruppe para-ständige Atom C-4 des substituierten Aromaten als Bezugsatom für die Winkelbestimmung gewählt.

Die ${ }^{13} \mathrm{C}$-chemischen Verschiebungen der Carbonyl-Kohlenstoffatome waren dafür nicht verwendbar, da eine durch Torsion bewirkte Resonanzminderung der Carbonylgruppe mit dem substituierten Aromaten eine Verstärkung der Resonanz mit dem zweiten, unsubstituierten Phenylring zur Folge hat; außerdem kann die in den 2-Hydroxy- 
ketonen 2 mögliche Wasserstoffbrückenbindung zwischen Hydroxy- und Carbonylfunktion zu nicht abschätzbaren Änderungen in der Elektronendichte am Carbonylkohlenstoff führen.

Nachdem bekannt ist, daß die chemische Verschiebung des para-Kohlenstoffatoms in monosubstituierten Benzolen eine lineare Beziehung zu den Hammettschen $\sigma$-Werten aufweist ${ }^{\text {9) }}$, war zu erwarten, daß die chemische Verschiebung des von uns gewählten Atoms C-4 die Summe aller Substituenteneinflüsse widerspiegelt. Um die alleinige Beeinflussung der chemischen Verschiebung von C-4 durch die tordierte CO-Gruppe zu erhalten, mußten die in Tab. 5 aufgeführten ${ }^{13} \mathrm{C}$-NMR-spektroskopischen Daten für C4 zunächst mit den ${ }^{13} \mathrm{C}$-Inkrementen der noch vorhandenen Substituenten korrigiert werden. Hierzu wurden einmal die von Doucet et al. ${ }^{10)}$ an para-disubstituierten Benzolen $\mathrm{X}-\mathrm{C}_{6} \mathrm{H}_{4}-\mathrm{Y}$ bestimmten Verschiebungskonstanten $S_{\mathrm{i}, \mathrm{Y}}^{\mathrm{X}}$ verwendet, die am ipsoKohlenstoffatom $\mathrm{C}-4$ bei Substitution von $\mathrm{H}$ durch $\mathrm{Y}$ im Aromaten $\mathrm{C}_{6} \mathrm{H}_{5} \mathrm{X}$ resultieren

$$
S_{\mathrm{i}, \mathrm{OH}}^{\mathrm{COPh}}=+28.90 \mathrm{ppm}, S_{\mathrm{i}, \mathrm{CH}_{3}}^{\mathrm{COPh}}=+11.75 \mathrm{ppm} \text { und } S_{\mathrm{i}, \mathrm{Cl}}^{\mathrm{COPh}}=+6.70 \mathrm{ppm}
$$

und zum anderen die Inkremente der weiteren meta-ständigen Substituenten ${ }^{11)}$.

Tab. 5. ${ }^{13}$ C-NMR-spektroskopische Daten der (2-Hydroxyphenyl)- 2 und (4-Hydroxyphenyl)phenylketone 3

\begin{tabular}{|c|c|c|c|c|c|c|c|c|c|c|}
\hline Keton & $2 \frac{a}{2}$ & $3 \mathrm{a}$ & $\underline{2 b}$ & $\stackrel{2 b^{\prime}}{=}=$ & $\underline{3 b}$ & $\stackrel{2 c}{=}$ & $2 \mathrm{c}_{\underline{z}}^{\prime}$ & $3 \mathrm{c}$ & $2 \mathrm{~d}$ & $3 \mathrm{~d}$ \\
\hline $\mathbf{R}$ & $\mathrm{OH}$ & $\mathrm{H}$ & $\mathrm{OH}$ & $\mathrm{OH}$ & $\mathrm{CH}_{3}$ & $\mathrm{OH}$ & OtI & $\mathrm{cl}$ & OH & $\mathrm{CH}_{3}$ \\
\hline $\mathrm{R}^{\prime}$ & $\mathrm{H}$ & $\mathrm{OH}$ & $\mathrm{CH}_{3}$ & $\mathrm{H}$ & $\mathrm{OH}$ & $\mathrm{cl}$ & H & $\mathrm{OH}$ & $\mathrm{CH}_{3}$ & OtI \\
\hline $\mathrm{R}^{\prime \prime}$ & y & $\mathrm{H}$ & $\mathrm{H}$ & $\mathrm{CH}_{3}$ & $\mathrm{H}$ & H & $\mathrm{Cl}$ & H & $\mathrm{CH}_{3}$ & $\mathrm{CH}_{3}$ \\
\hline$c=0$ & 201.607 & 197.369 & 201.061 & 200.724 & 199.343 & 200.733 & 197.819 & 196.983 & 201.190 & 201.382 \\
\hline$c-1$ & 119.185 & 129.267 & 116.874 & 123.825 & 129.990 & 117.709 & 123.071 & 129.524 & 123.969 & 132.093 \\
\hline$c-2$ & 163.302 & 133.251 & 163.495 & 157.506 & 141.131 & 163.912 & 158.229 & 133.794 & 159.192 & 136.492 \\
\hline$c-3$ & 118.639 & 115.557 & 118.511 & 114.706 & 112.234 & 118.544 & 116.039 & 117.676 & 115.429 & 114.626 \\
\hline$c-4$ & 136.299 & 161.343 & 148.018 & 133.008 & 158.791 & 142.207 & 133.506 & 159.224 & 144.390 & 156.174 \\
\hline$c-5$ & 118.447 & 115.557 & 119.956 & 122.637 & 118.431 & 119.346 & 121.915 & 114.112 & 120.534 & 114.626 \\
\hline$c-6$ & 133.602 & 133.281 & 133.505 & 139.398 & 132.783 & 134.437 & 133.121 & 131.772 & 140.377 & 135.492 \\
\hline $2-\mathrm{CH}_{3}$ & & & & & 20.693 & & & & & 19.618 \\
\hline $4-\mathrm{CH}_{3}$ & & & 21.962 & & & & & & 22.441 & \\
\hline $6-\mathrm{CH}_{3}$ & & & & 21.753 & & & & & 21.576 & 19.618 \\
\hline
\end{tabular}

\begin{tabular}{|c|c|c|c|c|c|c|c|c|c|c|}
\hline $\begin{array}{l}\text { Korrektu } \\
\text { faktor } \mathrm{x} \\
\text { c-4 }\end{array}$ & & & & & & & & & & \\
\hline Lit. ${ }^{10)}$ & -1.60 & -28.90 & $\begin{array}{l}-11.75 \\
-1.50\end{array}$ & -1.60 & -28.90 & $\begin{array}{l}-6.70 \\
-1.60\end{array}$ & $\begin{array}{l}-1.60 \\
-1.0\end{array}$ & $\begin{array}{l}-28.90 \\
-1.0\end{array}$ & $\begin{array}{l}-11.75 \\
-1.60\end{array}$ & -28.90 \\
\hline
\end{tabular}

Korrigier-

ter wert

$\begin{array}{lllllllllll}\mathrm{C}-4 & 134.70 & 132.44 & 134.67 & 131.41 & 129.89 & 133.91 & 130.91 & 129.32 & 131.04 & 127.27\end{array}$

Chem. Ber. II5 (1982) 
Analog Lit. ${ }^{12)}$ haben wir dann mit den korrigierten Werten für die chemische Verschiebung von C-4 die Torsionswinkel $\Theta$ der Hydroxyketone 2 und 3 nach

$$
\Theta=\arccos \sqrt{\frac{\delta_{\mathrm{C}-4}^{\Theta}-\delta_{C-4}^{90}}{\delta_{\mathrm{C}-4}^{0}-\delta_{C-4}^{90}}}
$$

bestimmt, wobei die hochgestellten Indices $\Theta, 90$ und 0 jeweils die chemische Verschiebung der C-4-Atome bei dem Torsionswinkel $\Theta$, dem Winkel $90^{\circ}$ bzw. $0^{\circ}$ bedeuten.

In der Literatur wird lediglich (2-Hydroxyphenyl)phenylketon (2a) als Beispiel für eine Verbindung mit einer koplanaren Anordnung $\left(\Theta=0^{\circ}\right)$ angegeben ${ }^{8)}$, während ein Beispiel für eine Verbindung mit orthogonaler Anordnung $\left(\Theta=90^{\circ}\right)$ bislang fehlt. Wir haben daher für das sterisch am stärksten gehinderte (4-Hydroxy-2,6-dimethylphenyl)phenylketon (3d) eine solche orthogonale Anordnung mit einem Torsionswinkel von $\Theta$ $=90^{\circ}$ angenommen und aus den korrigierten $\delta$-Werten dieser beiden Verbindungen $2 \mathrm{a}$ und 3d

$$
\left(\delta_{\mathrm{C}-4}^{0}=134.70 \mathrm{ppm} \text { und } \delta_{\mathrm{C}-4}^{90}=127.27 \mathrm{ppm}, \delta_{\mathrm{C}-4}^{0}-\delta_{\mathrm{C}-4}^{90}=7.43 \mathrm{ppm}\right)
$$

die Torsionswinkel $\Theta$ der übrigen von uns untersuchten Hydroxyketone nach der oben angegebenen Formel berechnet (Tab. 6).

Tab. 6. Torsionswinkel $\odot$ der Hydroxyketone 2 und 3

\begin{tabular}{cccc}
\hline Keton & $\left(\delta_{\mathrm{C}-4}^{\Theta}-\delta_{\mathrm{C}-4}^{90}\right)$ & $\cos ^{2} \Theta$ & $\Theta$ \\
\hline $\mathbf{2 a}$ & 7.43 & 1 & $0^{\circ}$ Lit. $^{12)} 0^{\circ}$ \\
$\mathbf{3 a}$ & 5.17 & 0.696 & $34^{\circ}$ \\
$\mathbf{2 b}$ & 7.40 & 0.966 & $4^{\circ}$ \\
$\mathbf{2 b}$ & 4.14 & 0.557 & $42^{\circ}$ \\
$\mathbf{3 b}$ & 2.62 & 0.353 & $54^{\circ}$ \\
$\mathbf{2 c}$ & 6.64 & 0.894 & $19^{\circ}$ \\
$\mathbf{2 c}$ & 3.64 & 0.490 & $46^{\circ}$ \\
$\mathbf{3 c}$ & 2.05 & 0.276 & $58^{\circ}$ \\
$\mathbf{2 d}$ & 3.77 & 0.507 & $45^{\circ}$ Lit. ${ }^{12)} 43^{\circ}$ \\
$\mathbf{3 d}$ & 0 & 0 & $90^{\circ}$ \\
\hline
\end{tabular}

Die so bestimmten Winkel zeigen eine gute Übereinstimmung mit den wenigen bislang in der Literatur angegebenen Werten; so ist z. B. der Torsionswinkel $\Theta$ für $\mathbf{3 b}$ $\left(53.6^{\circ}\right)$ mit dem von (2-Methylphenyl)phenylketon $\left(55^{\circ}\right)^{13)}$ vergleichbar; der von uns für 2d ermittelte Wert $\left(44.6^{\circ}\right)$ stimmt gut mit dem Literaturwert ${ }^{12)}$ für $2 \mathrm{~d}$ (ca. $43^{\circ}$ ) überein.

\section{Diskussion der Ergebnisse}

Von den bekannten elektrophilen Aromatensubstitutionen wurden bis vor wenigen Jahren nur die Alkylierung und Sulfonierung als reversibel angesehen. Untersuchungen in neuerer Zeit zeigten jedoch, daß auch andere elektrophile Aromatensubstitutionen unter vergleichsweise milden Bedingungen reversibel verlaufen können ${ }^{14}$. Beispiele für die Reversibilität der Acylierung sind die präparativ interessanten Isomerisierungen von Acylaromaten ${ }^{15)}$, Transacylierungen bei Polymethylbenzolen ${ }^{16 b)}$, die Isomerisierung von Fluorfluorenonen mit Polyphosphorsäure ${ }^{16 c)}$ sowie die Fries-Umlagerung bei Verbindungen mit Alkylgruppen in ortho-Stellung zum abspaltenden Acylrest ${ }^{5,6 b)}$. Ein Be- 
weis für die Reversibilität der klassischen Friedel-Crafts-Acylierung mit $\mathrm{AlCl}_{3}$ wurde vor kurzem durch den Nachweis des Acylaustauschs erbracht ${ }^{16 a)}$.

Unsere vorliegenden Untersuchungen beweisen zweifelsfrei, daß die mit TFMS katalysierte Fries-Umlagerung in allen untersuchten Fällen reversibel ist und daß sie zu identischen Gleichgewichtsgemischen aus Arylestern und Hydroxyaryl-ketonen führt. Bei diesen Isomerisierungen muß zwischen den durch die Stabilität der verschiedenen Verbindungen bedingten Gleichgewichtskonzentrationen (thermodynamische Kontrolle) und den Umlagerungsgeschwindigkeiten (kinetische Kontrolle) unterschieden werden; ein Gesichtspunkt, dem bei mechanistischen Untersuchungen der Fries-Umlagerung nicht immer die ihm zukommende Bedeutung beigemessen wurde ${ }^{4)}$.

Bei den mit TFMS durchgeführten Fries- bzw. Retro-Fries-Umlagerungen ist es unseres Wissens erstmals gelungen, Gleichgewichtsgemische aus Arylestern und Hydroxyaryl-ketonen zu erhalten. Unterschiedliche Katalysatormengen (TFMS) beeinflussen nur die Umlagerungsgeschwindigkeit, wie ein Vergleich der Isomerisierungen mit 20 bzw. 50 mol-\% TFMS zeigt (Tab. 1). Demgegenüber hängt bei der Fries-Umlagerung mit Aluminiumhalogeniden die Produktverteilung sehr von der Katalysatormenge $\mathrm{ab}^{4,17)}$; entscheidend sind hierbei sicher Komplexierungen, deren Einfluß auf die Isomerenbildung nur schwer abschätzbar ist. Zur Diskussion der Stabilität der verschiedenen Verbindungen kann vereinfachend angenommen werden, daß die Stabilitäten bei den verschiedenen Estern 1a-d von den meta-Substituenten im Phenolteil wenig beeinflußt werden und vergleichbar sind. Die Produktverteilung anhand der aus den Tab. 1-4 zu entnehmenden Gleichgewichtskonzentrationen hängt daher hauptsächlich von der Stabilität der Hydroxyaryl-ketone ab, die über die Verdrillungswinkel $\Theta$ zwischen CO-Gruppe und Hydroxyaryl-Rest bestimmt werden kann. Mit zunehmender Größe von $\Theta$ nimmt die Mesomeriewechselwirkung zwischen CO-Gruppe und HydroxyarylRest $\mathrm{ab}$, so daß unter den angewandten Äquilibrierungsbedingungen Hydroxyarylketone mit $\Theta>35^{\circ}$ offensichtlich instabiler sind als die Arylester.

Die Ausbildung intramolekularer $\mathrm{H}$-Brückenbindungen bei den ortho-Hydroxyarylketonen erzwingt in starkem Maße eine coplanare Anordnung von CO-Gruppe und Hydroxyaryl-Rest und bewirkt dadurch eine Stabilisierung dieser Verbindungen, wie Vergleiche zwischen $\mathbf{2 a}$ und $\mathbf{3 a}$, zwischen $\mathbf{2 b}, \mathbf{2} \mathbf{b}^{\prime}$ und $\mathbf{3 b}$ sowie zwischen $\mathbf{2 c}, \mathbf{2} \mathbf{c}^{\prime}$ und $\mathbf{3 c}$ zeigen. Wie groß dabei der Stabilisierungsbeitrag durch intramolekulare $\mathrm{H}$-Brücken selbst ist, kann nicht gesagt werden.

Bei allen untersuchten Hydroxyarylphenyl-ketonen sollte die Verdrillung des nicht substituierten Phenylrestes zur Keto-Gruppe vergleichbar sein und damit auch der Beitrag zur Stabilisierung der Ketone.

Die Isomerisierungsgeschwindigkeiten hängen außer von der Reaktionstemperatur und Säurekonzentration auch von der Struktur des Substrats ab. Unsere Versuchsergebnisse bestätigen, daß für die Deacylierungsgeschwindigkeit elektronische und sterische Einflüsse, die die Bildung der Wheland-Zwischenverbindung begünstigen, bedeutungsvoll sind ${ }^{14)}$. Unter Gleichgewichtsbedingungen (hohe Temperatur und lange Reaktionszeiten) können die besonders rasch isomerisierenden Hydroxyaryl-ketone mit ortho-Substituenten zur CO-Gruppe nicht mehr nachgewiesen werden (s. Tab. 2). Nur in wenigen Fällen haben wir die Produktverteilungen nach kurzen Reaktionszeiten, die 
Aussagen über die Umlagerungsgeschwindigkeiten der verschiedenen Isomeren erlauben würden, ermittelt (Tab. 2), wobei die rasche Umlagerung des para-Hydroxyarylketons $\mathbf{3 b}$ in den Ester $\mathbf{1 b}$ und nur wenig ortho-Hydroxyaryl-keton $\mathbf{2 b}$ überraschte.

Die Befunde der mit Brønsted-Säuren katalysierten Phenolacylierungen lassen sich wie folgt interpretieren: Für die Acylierungsgeschwindigkeit (kinetische Kontrolle) läßt sich folgende Reihe aufstellen: Arylester $>$ para-Hydroxyaryl-keton > orthoHydroxyaryl-keton, wie die ausschließliche Bildung von Arylestern in siedendem Methylenchlorid ${ }^{18)}$ und die überwiegende Bildung von para-Hydroxyaryl-ketonen bei Temperaturen bis $100^{\circ} \mathrm{C}$ mit Fluorwasserstoff ${ }^{19 a}$ bzw. Bortrifluorid ${ }^{19 b)}$ belegen. orthoHydroxyaryl-ketone entstehen erst bei höheren Temperaturen oder längeren Reaktionszeiten. Alle Befunde der säurekatalysierten Umlagerungen sprechen für einen dissoziativen und gegen einen Synchron-Mechanismus, was insbesondere durch die Isomerisierung $\mathbf{3 b} \rightarrow \mathbf{1 b} \rightarrow \mathbf{2 b}$ bestätigt wird. Auch für die mit Lewis-Säuren katalysierten Umlagerungen erscheint ein dissoziativer Mechanismus wahrscheinlicher ${ }^{17)}$. Bei langen Reaktionszeiten und hohen Temperaturen (thermodynamische Kontrolle) ist die Isomerenverteilung durch die Stabilität der Produkte bestimmt, da die Reversibilität der Acylierung für alle Isomeren nachgewiesen werden konnte.

Wir danken dem Fonds der Chemischen Industrie und der Deutschen Forschungsgemeinschaft für die Unterstützung dieser Arbeit.

\section{Experimenteller Teil}

${ }^{13}$ C-NMR-Spektren: Gerät HX (25.67 MHz) der Firma Bruker; $\delta$ (ppm), bezogen auf TMS als int. Standard.

GC/MS-Spektren: nach Blum und Richter ${ }^{20)}$ modifiziertes Finnigan-GC/MS-System mit Carlo-Erba Fractovap 2151 AC-Spezial-Gaschromatograph der Firma Brechbühler AG, Urdorf, mit Doppelsplitter und Septum-freier Direkteinspritzung nach Grob und Grob, jr. ${ }^{21)}$ direkt gekoppelt mit einem Finnigan 4023 Massenspektrometer mit Incos-Datensystem 2300. Trennkapillare $(20 \mathrm{~m}$, Emulphor 0 ) eigener Fertigung.

Gaschromatographie: a) Geräte 5700 A und 5710 A der Firma Hewlett-Packard mit Flammenionisationsdetektor (FID), Integratoren: Varian Aerograph 477 und Spectra-Physics „Minigrator". Trägergas: $30 \mathrm{ml} / \mathrm{min}$ Reinstickstoff. Glassäulen: 1) $2.3 \mathrm{~m} \times 2 \mathrm{~mm}$, Phase OV 25/5\% auf Gaschrom Q, 2) $2.3 \mathrm{~m} \times 2 \mathrm{~mm}$, Phase OV $17 / 10 \%$ auf Chromosorb W. Temperaturprogramm $100-300^{\circ} \mathrm{C}, 16^{\circ} / \mathrm{min}$, dann $8 \mathrm{~min}$ bei $300^{\circ} \mathrm{C}$. b) Carlo Erba Fractovap GI mit FID, Integrator: Spectra-Physics „Minigrator“. Trägergas: 0.7 bar Helium. Säule: Kapillare, 20 m, Phase SE 52. Temperaturprogramm: $40-250^{\circ} \mathrm{C}, 3^{\circ} / \mathrm{min}$, dann $10 \mathrm{~min}$ bei $250^{\circ} \mathrm{C}$.

Präparative Mitteldruck-Säulenchromatographie: nach Glatz ${ }^{22)}$ mit Lewa-Kolbenpumpe Typ FL 1 der Firma Ott, Pulsationsdämpfer eigener Fertigung, UV-Detektor der Firma Gilson. Kieselgelsäule $(250 \times 24 \mathrm{~mm})$ mit LiChroPrep Si $60(15-25 \mu)$ der Firma Merck. Theoretische Bodenzahl $N=7300$.

Thermostat: Heizbad mit Siliconöl P 300 der Firma Bayer, Meßfühler PT 100, Proportionalregler, Tauchsieder, Rührer und Einsatz zur Halterung für die Ampullen. 
Allgemeines: Alle Isomerisierungen wurden unter einer schwachen Stickstoffatmosphäre durchgeführt. Hierzu wurden die Lösungen der Substrate und Trifluormethansulfonsäure (TFMS) in absol. 1,2-Dichlorethan (DCE) im Stickstoffkasten in ausgeheizte und evakuierte 30ml-Glasampullen (aus Duran 50) eingewogen, die anschließend mit einem durchbohrten Stopfen mit Glashahn verschlossen und auf $0^{\circ} \mathrm{C}$ abgekühlt wurden. Nach ca. 10 s Evakuieren der Ampullen über den Hahn an der Wasserstrahlpumpe über einen dazwischengeschalteten Phosphorpentoxid-Trockenturm wurden die Ampullen abgeschmolzen und zur Isomerisierung bis zum eigenen Flüssigkeitsspiegel in das Siliconbad getaucht. Nach beendeter Reaktion und Abkühlen an der Luft auf Raumtemp. wurden die Lösungen mit dreimal $2 \mathrm{ml}$ DCE aus der geöffneten Ampulle herausgespült und mit $10 \mathrm{ml}$ einer $0.1 \mathrm{M}$ wäßrigen Natriumhydrogencarbonat-Lösung sowie der jeweiligen Standardlösung kräftig ausgeschüttelt. Die obere wäßr. Phase wurde einmal mit $5 \mathrm{ml}$ DCE und zweimal mit $2 \mathrm{ml}$ DCE extrahiert. Dann wurden alle organischen Phasen vereinigt und bei $40^{\circ} \mathrm{C} \mathrm{i}$. Wasserstrahlvak. im Rotationsverdampfer eingeengt. Der Rückstand wurde mit $3 \mathrm{ml}$ DCE aufgenommen, die Lösung mit $5 \mathrm{~g}$ Natriumsulfat getrocknet und gaschromatographisch analysiert.

Die angegebenen Ausbeuten sind auf den jeweiligen inneren Standard bezogen und wurden in Vergleichsmessungen mit authentischen Substanzen geeicht. Sie stellen Mittelwerte aus 3 Bestimmungen dar (Abweichung $\pm 0.5 \%$ ).

Ansätze: Es wurden jeweils $12.0 \mathrm{~g}$ einer Lösung von $1.0 \mathrm{mmol}$ Ester oder Hydroxyaryl-keton in absol. DCE mit $1.0 \mathrm{~g}$ einer Lösung von 2-20 mmol TFMS in absol. DCE isomerisiert bzw. mit $2.5 \mathrm{~g}$ einer Lösung von $50 \mathrm{mmol}$ TFMS in DCE.

Zu Tab. 1: Eingesetzt wurden jeweils $198.22 \mathrm{mg}$ (1.0 mmol) 1a, 2a oder 3a, $302.33 \mathrm{mg}$ (1.0 mmol) 4 oder $5 \mathrm{mit} 3.0 \mathrm{mg}(0.020 \mathrm{mmol}), 30.0 \mathrm{mg}(0.20 \mathrm{mmol})$ oder $75.0 \mathrm{mg}(0.50 \mathrm{mmol})$ TFMS in absol. DCE und als Standard Benzoin. Die Umsetzungen mit 50 mol- $\%$ TFMS ergaben nach 24 h: a) mit 4: 34\% 2a, 38\% Benzoesäure und 1\% Phenol; b) mit 5: 9\% 3a, 46\% Benzoesäure und 3\% Phenol.

Zu Tab. 2: Eingesetzt wurden jeweils $212.25 \mathrm{mg}(1.0 \mathrm{mmol}) \mathbf{1 b}, \mathbf{2 b}, \mathbf{2} \mathbf{b}^{\prime}$ oder $\mathbf{3 b}$ mit $15.0 \mathrm{mg}$ $(0.10 \mathrm{mmol})$ TFMS in absol. DCE und als Standard Bis(4-methoxyphenyl)keton.

Zu Tab. 3: Eingesetzt wurden jeweils $232.67 \mathrm{mg}(1.0 \mathrm{mmol}) 1 \mathrm{c}, 2 \mathrm{c}, 2 \mathrm{c}^{\prime}$ oder $3 \mathrm{c}$ mit $7.5 \mathrm{mg}$ $(0.050 \mathrm{mmol}), 15.0 \mathrm{mg}(0.10 \mathrm{mmol})$ oder $30.0 \mathrm{mg}(0.20 \mathrm{mmol})$ TFMS in absol. DCE und als Standard 4-Chlorbenzoesäure-4-chlorphenylester.

$\mathrm{Zu} \mathrm{Tab.} \mathrm{4:} \mathrm{Eingesetzt} \mathrm{wurden} \mathrm{jeweils} 226.28 \mathrm{mg}(1.0 \mathrm{mmol}) \mathbf{1 d}, 2 \mathrm{~d}$ oder $3 \mathrm{~d}$ mit $7.5 \mathrm{mg}(0.050$ mmol), $15.0 \mathrm{mg}(0.10 \mathrm{mmol}), 22.50 \mathrm{mg}(0.15 \mathrm{mmol})$ oder $45.0 \mathrm{mg}(0.30 \mathrm{mmol})$ TFMS in absol. DCE und als Standard (4-Hydroxy-3,5-dimethylphenyl)phenylketon.

Darstellung der Ausgangsverbindungen: Nach Lit. ${ }^{18)}$ : Phenylbenzoat (1a), Schmp. $70^{\circ} \mathrm{C}$ (Lit. 23) $71^{\circ} \mathrm{C}$ ), (3-Methylphenyl)benzoat (1b), Schmp. $55^{\circ} \mathrm{C}$ (Lit. ${ }^{23)} 55-56^{\circ} \mathrm{C}$ ), (3-Chlorphenyl)benzoat (1c), Schmp. $72^{\circ} \mathrm{C}$ (Lit. ${ }^{25)} 71-72^{\circ} \mathrm{C}$ ) und (3,5-Dimethylphenyl)benzoat (1d), Sdp. $179-180^{\circ} \mathrm{C} / 10$ Torr (Lit. ${ }^{18)}$ Schmp. $23^{\circ} \mathrm{C}$ ).

(2-Hydroxyphenyl)phenylketon (2a): Aus 2-Methoxybenzoesäure ${ }^{26)}$ nach Lit. ${ }^{27)} \rightarrow$ 2-Methoxybenzoylchlorid nach Lit. ${ }^{28)} \rightarrow 2$ a, Schmp. $39^{\circ} \mathrm{C}$ (Lit. ${ }^{23 \mathrm{c})} 39^{\circ} \mathrm{C}$ ).

(2-Hydroxy-4-methylphenyl)phenylketon (2b): Aus 2-Methoxy-4-methyibenzoesäure ${ }^{29)}$ nach Lit. ${ }^{30)} \rightarrow$ 2-Methoxy-4-methylbenzoylchlorid nach Lit. ${ }^{28)} \rightarrow 2$ b, Schmp. $61^{\circ} \mathrm{C}$ (Lit. ${ }^{31)}$ $\left.60-61^{\circ} \mathrm{C}\right)$.

(4-Chlor-2-hydroxyphenyl)phenylketon (2c): Aus 2-Amino-4-chlortoluol nach Lit. ${ }^{34)} \rightarrow 4$ Chlor-2-hydroxytoluol nach Lit. ${ }^{32)} \rightarrow$ 4-Chlor-2-methoxytoluol nach Lit. ${ }^{35)} \rightarrow$ 4-Chlor-2-meth- 
oxybenzoesäure nach Lit. ${ }^{36)} \rightarrow$ 4-Chlor-2-methoxybenzoylchlorid nach Lit. ${ }^{28)} \rightarrow 2 \mathrm{c}$, Schmp. $74^{\circ} \mathrm{C}$ (Lit. ${ }^{31)} 72-74^{\circ} \mathrm{C}$ ).

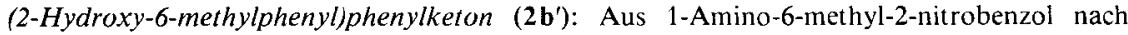
Lit. ${ }^{33)} \rightarrow$ 1-Brom-6-methyl-2-nitrobenzol nach Lit. ${ }^{37)} \rightarrow$ 2-Amino-1-brom-6-methylbenzol nach Lit. ${ }^{34)} \rightarrow$ 1-Brom-2-hydroxy-6-methylbenzol nach Lit. ${ }^{32)} \rightarrow$ 1-Brom-2-methoxy-6-methylbenzol nach Lit. ${ }^{38)} \rightarrow$ 2-Methoxy-6-methylbenzoesäure nach Lit . ${ }^{36)} \rightarrow$ 2-Methoxy-6-methylbenzoylchlorid $\rightarrow$ (2-Methoxy-6-methylphenyl)phenylketon: Zu der etherischen Lösung von Phenylmagnesiumbromid aus $1.73 \mathrm{~g}(11.0 \mathrm{mmol})$ Brombenzol und $0.27 \mathrm{~g}(11.0 \mathrm{mmol})$ Magnesium gibt man bei $0^{\circ} \mathrm{C} 1.01 \mathrm{~g}(5.5 \mathrm{mmol})$ Cadmiumchlorid, rührt $30 \mathrm{~min}$ unter Sieden, tropft bei Raumternp. $1.85 \mathrm{~g}$ (10.0 mmol) 2-Methoxy-6-methylbenzoylchiorid zu, rührt $4 \mathrm{~h}$ bei Raumtemp. und 30 min unter Rückfluß. Nach Aufarbeiten mit Eiswasser/Salzsäure wird mit Ether extrahiert, die etherische Lösung mit Natriumcarbonat neutralisiert und getrocknet. Nach Abdestillieren des Ethers wird aus dem Rückstand das Produkt durch Mitteldruck-Chromatographie mit tiefsiedendem Petrolether/Essigsäure-ethylester (3:1) abgetrennt. Ausb. $1.50 \mathrm{~g}(67 \%)$.

$2 b^{\prime}$ : Die Lösung von $1.50 \mathrm{~g}(6.7 \mathrm{mmol})$ (2-Methoxy-6-methylphenyl)phenyiketon (s. vorstehend) und $2.01 \mathrm{~g}$ ( $13.4 \mathrm{mmol})$ Natriumiodid in $30.0 \mathrm{~g}$ Acetonitril wird mit $1.46 \mathrm{~g}$ (13.4 mmol) Chlortrimethylsilan versetzt und im Glasautoklaven $24 \mathrm{~h}$ bei $130^{\circ} \mathrm{C}$ umgesetzt. Nach Abkühlen wird mit Eiswasser/Salzsäure, gesättigter wäßr. Natriumthiosulfatlösung und gesättigter Natriumchloridlösung gewaschen und das Acetonitril im Rotationsverdampfer entfernt. Aus dem Rückstand wird $\mathbf{2} \mathbf{b}^{\prime}$ durch Mitteldruck-Chromatographie mit tiefsiedendem Petrolether/Essigsäure-ethylester $(9: 1)$ abgetrennt. Ausb. $0.214 \mathrm{~g}(15 \%)$, Schmp. $73^{\circ} \mathrm{C} .-\mathrm{GC} / \mathrm{MS}: \mathrm{MH}^{+} m / e=$ $213, \mathrm{C}_{6} \mathrm{H}_{5} \mathrm{CO}^{+} 105,\left(\mathrm{CH}_{3}\right)(\mathrm{HO}) \mathrm{C}_{6} \mathrm{H}_{3} \mathrm{CO}^{+} 135$.

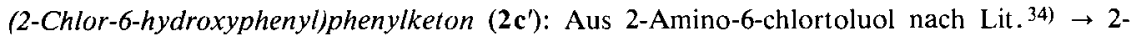
Chlor-6-hydroxytoluol nach Lit . ${ }^{32)} \rightarrow$ 2-Chlor-6-methoxytoluol nach Lit. ${ }^{35)} \rightarrow$ 2-Chlor-6-methoxybenzoesäure $\rightarrow$ 2-Chlor-6-methoxybenzoylchlorid: Aus $3.72 \mathrm{~g} \mathrm{(19.9} \mathrm{mmol)} \mathrm{2-Chlor-6-meth-}$ oxybenzoesäure und $4.75 \mathrm{~g}$ (21.9 mmol) Phosphorpentachlorid nach Lit. ${ }^{36)}$ Ausb. $3.68 \mathrm{~g}(90 \%)$, Sdp. $100^{\circ} \mathrm{C} / 10^{-1}$ Torr. (2-Chlor-6-methoxyphenyl)phenylketon: Wie vorstehend (s. Darstellung von (2-Methoxy-6-methylphenyl)phenylketon) aus der etherischen Lösung von Phenylmagnesiumbromid aus $8.79 \mathrm{~g}(56.0 \mathrm{mmol})$ Brombenzol und $1.36 \mathrm{~g}(56.0 \mathrm{mmol})$ Magnesium, $5.13 \mathrm{~g} \mathrm{(28.0}$ mmol) Cadmiumchlorid und $10.44 \mathrm{~g}(51.0 \mathrm{mmol}) 2$-Chlor-6-methoxybenzoylchlorid nach Aufarbeiten $5.71 \mathrm{~g} \mathrm{(46 \% ).} \mathrm{-} 2 \mathrm{c}^{\prime}$ : analog $2 \mathrm{~b}^{\prime}$ (s. vorstehend) aus $5.71 \mathrm{~g}(23.0 \mathrm{mmol}$ ) (2-Chlor-6-methoxyphenyl)phenylketon, $4.97 \mathrm{~g}(46.0 \mathrm{mmol})$ Chlortrimethylsilan und $6.85 \mathrm{~g}(46.0 \mathrm{mmol})$ Natriumiodid in $120 \mathrm{ml}$ Acetonitril nach Aufarbeiten Ausb. $1.5 \mathrm{~g} \mathrm{(36 \% ),} \mathrm{Schmp.} 105^{\circ} \mathrm{C}$.

(4-Hydroxyphenyl)phenylketon (3a): Aus 4-Nitrobenzoylchlorid nach Lit. ${ }^{39)} \rightarrow$ (4-Nitrophenyl)phenylketon nach Lit. ${ }^{37)} \rightarrow\left(4\right.$-Aminophenyl)phenylketon nach Lit. ${ }^{40)} \rightarrow 3 \mathrm{a}$, Schmp. $133^{\circ} \mathrm{C}$ (Lit. 24) $135^{\circ} \mathrm{C}$ ).

(4-Hydroxy-2-methylphenyl)phenylketon (3b): Aus (3-Methylphenyl)benzoat (1b) nach Lit. 5) $\rightarrow$ 3b, Schmp. $130^{\circ} \mathrm{C}$ (Lit. ${ }^{41)} 129^{\circ} \mathrm{C}$ ).

(2-Chlor-4-hydroxyphenyl)phenylketon (3c): Aus 2-Chlor-4-nitrobenzoesäure nach Lit. ${ }^{36)} \rightarrow$ 2-Chlor-4-nitrobenzoylchlorid nach Lit. ${ }^{28)} \rightarrow(2$-Chlor-4-nitrophenyl)phenylketon: Aus $3.75 \mathrm{~g}$ (17.0 mmol) 2-Chlor-4-nitrobenzoylchlorid, $13.31 \mathrm{~g} \mathrm{(170.0} \mathrm{mmol)} \mathrm{Benzol} \mathrm{und} 3.79 \mathrm{~g} \mathrm{(28.0} \mathrm{mmol)}$ Aluminiumchlorid Ausb. $4.25 \mathrm{~g}(96 \%)$, Schmp. $96^{\circ} \mathrm{C}$. - (4-Amino-2-chlorphenyl)phenylketon: $\mathrm{Zu}$ der Lösung von $3.0 \mathrm{~g}(11.5 \mathrm{mmol})$ (2-Chlor-4-nitrophenyl)phenylketon in $60 \mathrm{ml}$ konz. Salzsäure und $60 \mathrm{ml}$ Isopropylalkohol werden in der Siedehitze portionsweise unter Rühren $10.0 \mathrm{~g}(84.3$ mmol) Zinn gegeben. Anschließend wird $1 \mathrm{~h}$ zum Sieden erhitzt, nach Erkalten mit konz. Natronlauge neutralisiert, mit Ether unter Zusatz von wenig Benzol extrahiert und der Extrakt nach Trocknen eingeengt. Ausb. $2.25 \mathrm{~g} \mathrm{(85 \% ),} \mathrm{Schmp.} 143^{\circ} \mathrm{C}$. - 3c: $2.25 \mathrm{~g}(10.0 \mathrm{mmol})$ (4-Amino-2- 
chlorphenyl)phenylketon werden mit $0.68 \mathrm{~g}(10.0 \mathrm{mmol})$ Natriumnitrit nach Lit. ${ }^{34)}$ diazotiert. Ausb. $1.5 \mathrm{~g}(66 \%)$, Schmp. $115^{\circ} \mathrm{C}$.

$$
\mathrm{C}_{13} \mathrm{H}_{9} \mathrm{ClO}_{2} \text { (232.7) Ber. C } 67.11 \mathrm{H} 3.90 \mathrm{Cl} 15.24 \text { Gef. C } 67.01 \mathrm{H} 3.91 \mathrm{Cl} 14.96
$$

(2-Hydroxy-4,6-dimethylphenyl)phenylketon (2d): Aus (3,5-Dimethylphenyl)benzoat (1 d) nach Lit. ${ }^{5)}$, Schmp. $140^{\circ} \mathrm{C}$ (Lit. ${ }^{42)} 140^{\circ} \mathrm{C}$ ).

(4-Hydroxy-2,6-dimethylphenyl)phenylketon (3d): Aus 1-Brom-4-hydroxy-2,6-dimethylbenzol nach Lit. ${ }^{32)} \rightarrow$ 1-Brom-4-benzyloxy-2,6-dimethylbenzol nach Lit. ${ }^{38)} \rightarrow$ 4-Benzyloxy-2,6-dimethylbenzoesäure nach Lit. ${ }^{38)} \rightarrow$ 4-Benzyloxy-2,6-dimethylbenzoylchlorid: Aus $5.7 \mathrm{~g}(22.0$ mmol) 4-Benzyloxy-2,6-dimethylbenzoesäure und $8.46 \mathrm{~g}(71.0 \mathrm{mmol})$ Thionylchlorid Ausb. $5.17 \mathrm{~g}(85 \%)$, Sdp. $145^{\circ} \mathrm{C} / 10^{-2}$ Torr. - (4-Benzyloxy-2,6-dimethylphenyl)phenylketon: Analog der Darstellung von (2-Methoxy-6-methylphenyl)phenylketon (s. vorstehend unter $\mathbf{2} \mathbf{b}^{\prime}$ ) aus der etherischen Lösung von Phenylmagnesiumbromid (aus $3.25 \mathrm{~g}, 20.7 \mathrm{mmol}$, Brombenzol und $0.50 \mathrm{~g}, 20.7 \mathrm{mmol}$, Magnesium), $1.895 \mathrm{~g}$ (10.35 mmol) Cadmiumchlorid und $5.17 \mathrm{~g}(18.8 \mathrm{mmol})$

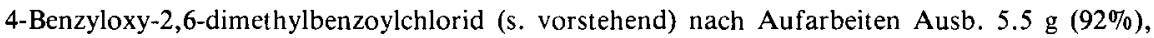

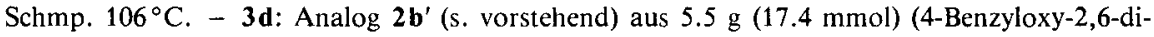
methylphenyl)phenylketon, $3.8 \mathrm{~g} \mathrm{(35.0} \mathrm{mmol)} \mathrm{Chlortrimethylsilan} \mathrm{und} 5.15 \mathrm{~g} \mathrm{(35.0} \mathrm{mmol)} \mathrm{Natri-}$ umiodid in $120 \mathrm{ml}$ Acetonitril nach Aufarbeiten Ausb. $2.38 \mathrm{~g}(60 \%)$, Sdp. $153-155^{\circ} \mathrm{C} / 10^{-3}$ Torr, Schmp. $115^{\circ} \mathrm{C}$.

$$
\mathrm{C}_{15} \mathrm{H}_{14} \mathrm{O}_{2} \text { (226.3) Ber. C } 79.62 \mathrm{H} 6.24 \text { Gef. C } 79.44 \mathrm{H} 6.22
$$

[2-(Benzoyloxy)phenyl]phenylketon (4) 43): Man läßt zu der Lösung von $2.62 \mathrm{~g}$ (13.22 mmol) (2Hydroxyphenyl)phenylketon (2a) in $15 \mathrm{ml}$ Pyridin unter Rühren bèi $0^{\circ} \mathrm{C} 1.86 \mathrm{~g}(13.22 \mathrm{mmol})$ Benzoylchlorid tropfen, rührt dann $30 \mathrm{~min}$ be $40^{\circ} \mathrm{C}$, filtriert und extrahiert das Filtrat nach Waschen mit Eiswasser mit Ether. Die etherischen Extrakte werden getrocknet und destilliert. Ausb. $3.31 \mathrm{~g}(83 \%)$, Sdp. $165-170^{\circ} \mathrm{C} / 10^{-2}$ Torr.

$$
\mathrm{C}_{20} \mathrm{H}_{14} \mathrm{O}_{3} \text { (302.3) Ber. C } 79.46 \mathrm{H} 4.67 \text { Gef. C } 79.34 \mathrm{H} 4.68
$$

[4-(Benzoyloxy)phenyl]phenylketon (5): Man läßt zu der Lösung von $0.70 \mathrm{~g}$ (4-Hydroxyphenyl)phenylketon (3a) in $3 \mathrm{ml}$ Pyridin unter Rühren bei Raumtemp. $0.50 \mathrm{~g}$ ( $3.5 \mathrm{mmol}$ ) Benzoylchlorid tropfen, läßt über Nacht stehen, saugt ab und versetzt das Filtrat mit Wasser. Der farblose Niederschlag wird abfiltriert, Ausb. $1.02 \mathrm{~g}(96 \%)$, Schmp. $112^{\circ} \mathrm{C}$ (Lit. ${ }^{44)} 113-114^{\circ} \mathrm{C}$ ).

1) 23. Mitteil.: Zur elektrophilen Aromatensubstitution; 22. Mitteil.: F. Effenberger, K. Huthmacher und M. Keil, Chem. Ber. 114, 1967 (1981).

2) R. Gutmann, Dissertation, Univ. Stuttgart 1981.

3) 3a) A. H. Blatt, Org. React. 1, $342(1942)$. - 3b) H. Henecka in Methoden der organischen Chemie (Houben-Weyl-Müller), Bd. VII/2a, S. 379, G. Thieme Verlag, Stuttgart 1973. - 3c) T. Laird in Comprehensive Organic Chemistry, Edit. J. F. Stoddard, Vol. 1, S. 1161, Pergamon Press, Oxford, New York, Toronto, Sidney, Paris, Frankfurt 1979.

4) M. J. S. Dewar und L. S. Hart, Tetrahedron 26, 973 (1970).

5) K.W. Rosenmund und W. Schnurr, Liebigs Ann. Chem. 460, 56 (1928).

6) 6a) $N . M$. Cullinane und B.F.R. Edwards, J. Chem. Soc. 1958, 434. - 6b) J. F. Miquel, P. Müller und N. P. Buu-Hoi, Bull. Soc. Chim. Fr. 1956, 633.

7) F. Effenberger, H. Klenk und P. L. Reiter, Angew. Chem. 85, 819 (1973); Angew. Chem., Int. Ed. Engl. 12, 775 (1973).

8) K. S. Dhami und J. B. Stothers, Tetrahedron Lett. 1964, 631.

9) H. Spiesecke und W. G. Schneider, J. Chem. Phys. 35, 731 (1961).

10) F. Membrey, B. Ancian und J.-P. Doucet, Org. Magn. Reson. 11, 580 (1978).

11) $M$. Hesse, $H$. Meier und $B$. Zeeh in Spektroskopische Methoden in der organischen Chemie, S. 236, G. Thieme Verlag, Stuttgart 1979. 
12) S. Miertuš und $O$. Kysel, J. Mol. Struct. 26, 163 (1975).

13) G. W. Buchanan, G. W. Montaudo und P. Finocchiaro, Can. J. Chem. 51, 1053 (1973).

14) F. Effenberger, Chem. Unserer Zeit 13, 87 (1979).

15) D. E. Pearson und C. A. Buehler, Synthesis 1971, 455.

16) 16a) A. D. Andreou, P. H. Gore und D. F. C. Morris, J. Chem. Soc., Chem. Commun. 1978, 271. - 16b) A. D. Andreou, R. V. Bulbulian und P. H. Gore, J. Chem. Res. (M) 1980, 3001; (S) 1980, 225. - 16c) I. Agranat, Y. Bentor und $Y u$-Shan Shih, J. Am. Chem. Soc. 99, 7068 (1977).

17) R. Martin, Bull. Soc. Chim. Fr. 1974, 983.

18) $F$. Effenberger und $H$. Klenk, Chem. Ber. 107, 175 (1974).

19) 19a) O. Dann und G. Mylius, Liebigs Ann. Chem. 587, 1 (1954). - 19b) K. Freudenberg und $K$. Weinges, Liebigs Ann. Chem. 590, 149 (1954).

20) W. Blum und W. J. Richter, J. Chromatogr. 132, 249 (1977).

21) K. Grob und K. Grob, jr., J. Chromatogr. 151, 311 (1978).

22) B. Glatz, Dissertation, Univ. Stuttgart 1976.

23) $R$. C. Weast, Handbook of Chemistry and Physics, 57 Ed., C-182, The Chemical Rubber Co., Cleveland, Ohio $1976-1977$.

24) Lit. 23), C-202.

25) W. J. Wohlleben, Ber. Dtsch. Chem. Ges. 42, 4371 (1909).

26) C. Graebe, Liebigs Ann. Chem. 340, 210 (1905).

27) E. Fischer und M. Slimmer, Ber. Dtsch. Chem. Ges. 36, 2585 (1903)

${ }^{28)}$ F. F. Blicke und O. J. Weinkauff, J. Am. Chem. Soc. 54, 1448 (1932).

29) C. Schall, Ber. Disch. Chem. Ges. 12, 825 (1879).

${ }^{30)} R$. Anschütz, H. Aschenberg, H. Kuckertz, F. Krone, K. Riepenkröger und C. Zerbe, Liebigs Ann. Chem. 442, 35 (1925).

31) P. Hrdlovič, D. Belluš und M. Lazár, Collect. Czech. Chem. Commun. 33, 61 (1968).

32) Autorenkollektiv, Organikum, 15. Aufl., S. 251, VEB Deutscher Verlag der Wissenschaften, Berlin 1976.

33) Lit. ${ }^{32)}$, S. 655 und 664.

34) Lit. ${ }^{32)}$, S. 655 und 660 .

35) F. Ullmann und L. Panchaud, Liebigs Ann. Chem. 350, 113 (1906).

36) C. Weygand und $G$. Hilgetag in Organisch-chemische Experimentierkunst, 4. Aufl., S. 247, J. Ambrosius Barth Verlag, Leipzig 1970.

37) Lit. 36), S. 573.

38) R. P. Barnes in Organic Syntheses, Edit. E. C. Horning, Coll. Vol. III, S. 555, J. Wiley and Sons, London 1955.

39) G. Schroeter, Ber. Dtsch. Chem. Ges, 42, 3360 (1909).

40) O. Doebner, Liebigs Ann. Chem. 210, 274 (1881).

41) E. H. Cox, J. Am. Chem. Soc. 49, 1028 (1927).

42) P. Hrdlovič, N. Schubertova, B. Arventiev und H. Wexler, Collect. Czech. Chem. Commun. 36, 1949 (1971).

43) F. Nahavandi, F. Razmara und M. P. Stevens, Tetrahedron Lett. 1973, 301.

44) C. R. Hauser und E. H. Man, J. Org. Chem. 17, 394 (1952). 